\title{
Systematic influences on the areas of peaks in gamma- ray spectra that have a large statistical uncertainty
}

M. Bruggeman ${ }^{a}$, S.M. Collins ${ }^{b}$, L. Done ${ }^{h}$, M. Đuraševićc, M.A. Duch ${ }^{d}$, A. Gudelis ${ }^{e}$, M. Hyža ${ }^{f}$, A. Jevremovićc,

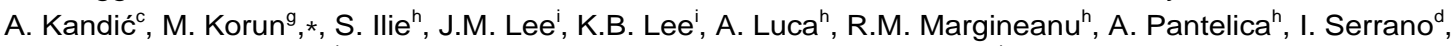

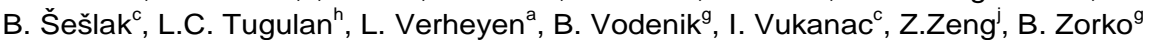

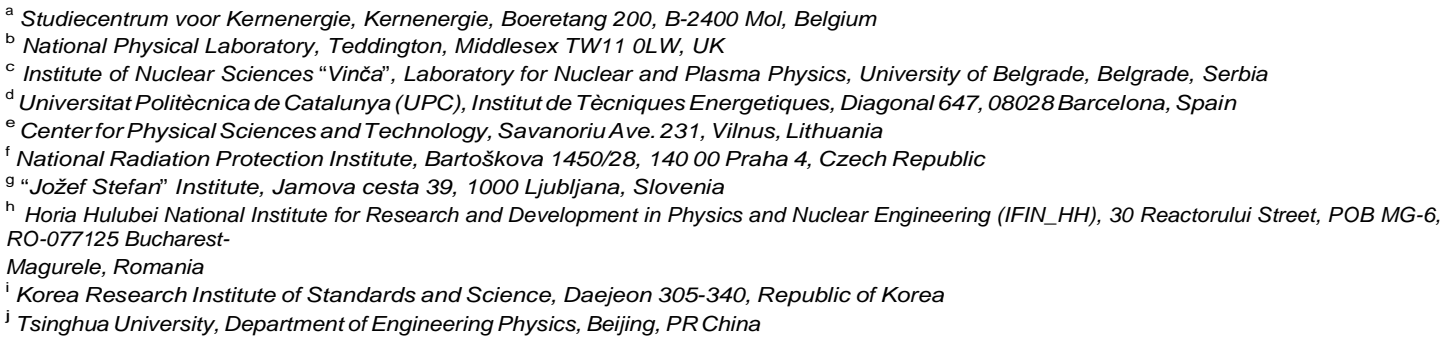

Highlights

The results of peak analysis programs were tested on a statistical basis.

Peak areas with a large statistical uncertainty were tested for their trueness.

The test was performed on five different programs for analyzing the spectra.

It was found that peak areas with a large statistical uncertainty are too large.

A way, how to calculate uncertainty-dependent correction factors is described.

\section{Keywords:}

Gamma-ray spectrometry, Systematic influence, Peak area, Relative uncertainty, Correction factors

Abstract:

A method is presented for calculating the expected number of counts in peaks that have a large relative peak- area uncertainty and appear in measured gamma-ray spectra. The method was applied to calculations of the correction factors for peaks occurring in the spectra of radon daughters. It was shown that the factors used for correcting the calculated peak areas to their expected values decrease with an increasing relative peak-area uncertainty. The accuracy of taking the systematic influence inducing the correction factors into account is given by the dispersion of the correction factors corresponding to specific peaks. It was shown that the highest accuracy is obtained in the peak analyses with the GammaVision and Gamma-W software.

\section{Introduction}

In gamma-ray spectra the information about the activities of the gamma-ray emitters is related to the number of counts registered in the peaks appearing in the spectra. Therefore, computerized gamma-ray spectrometry analyses, aimed at calculating the activities from spectra measured with a high-resolution gamma-ray detector, evaluate these numbers of counts, i.e., the peak areas. There are two basic methods for locating the peaks in the spectra: the nuclide-library-directed method and the peak-search method (Gilmore, 2008). The nuclide-library-directed method only checks for the presence of peaks at energies listed in the library, whereas the peak-search method scans the whole spectrum for the presence of peaks. Here, regions in the spectra that have a width comparable with the width of a peak and comprising more counts than neighbouring spectral regions are searched for. The location of the peak is determined on the basis of the statistical significance of the excess number of counts. If the statistical significance exceeds a predetermined value, the excess number of counts is attributed to a peak; otherwise it is disregarded as a statistical fluctuation of the continuous spectral background. Since the neighbouring regions are subject to statistical fluctuations too, influences of the systematic effects on the peak areas can occur. These are more expressive when the statistical significance of the peak is smaller, i.e., when the relative uncertainty of its area is larger.

To check the peak-analysis results for influences of systematic effects, a study was performed on a set of 35 spectra acquired with a source containing 226Ra that was displaced from the detector, to minimize the influence of coincidence-summing effects, under repeatable conditions (Korun et al., 2015). In these spectra many peaks having a wide range of expressivenesses appear. A comparatively large number of spectra were used in the study since inexpressive peaks cannot be located in all the spectra, even if the limit significance value was set low. To extend the study to peaks that are located with a small probability, a large set of spectra was analysed. Since the number of counts in all the peaks corresponds to the same activity, in the absence of systematic effects the quantity $\alpha(E)=n p(E)$ / $p(E) / \varepsilon S(E)$, where $n p(E), p(E)$ and $\varepsilon S(E)$ denote the peak area, the in- tensity and a supposed peak counting efficiency for a gamma ray with energy $E$, respectively, smoothly depends on the energy. Its energy dependence is 
given by $\varepsilon S(E) / \varepsilon(E)$, where $\varepsilon(E)$ denotes the true counting efficiency. As the systematic influences are not expected to appear in the peak areas of strong peaks, the energy dependence can be obtained at energies of $295 \mathrm{keV}, 352 \mathrm{keV}$, $609 \mathrm{keV}, 1120 \mathrm{keV}, 1765 \mathrm{keV}$ and $2204 \mathrm{keV}$, where the most distinct peaks appear in the spectra. At any other energy $E^{\prime}$ the quantity $\alpha\left(E^{\prime}\right)$ can be obtained by interpolation and with it the expected peak area $n p\left(E^{\prime}\right)=\alpha\left(E^{\prime}\right) p\left(E^{\prime}\right)$ $\varepsilon S\left(E^{\prime}\right)$. The peak-specific correction factor for the peak area reported by the peak-analysis program is then $C\left(E^{\prime}\right)=$ $n p\left(E^{\prime}\right) / n p C\left(E^{\prime}\right)$, where $n p C\left(E^{\prime}\right)$ denotes the area of the peak appearing at the energy $E^{\prime}$ in the peak-analysis results. The study has shown that large systematic influences occur at relative peak-area uncertainties in excess of $40 \%$. However, as the peak analysis was performed using a homemade procedure comprising three different peak-analysis methods (Korun et al., 2008), doubt has arisen about the relevance of the findings for the broader community engaged in gamma-ray spectroscopic measurements. To gain an overview about the significance of the conclusions of the study an action within the Low-Level Working Group of the International Committee for Radionuclide Metrology was performed where interested laboratories parti- cipated by performing peak analyses of the set of 35 spectra and reporting the results of the analyses. The laboratories were asked to analyse a set of 102 peaks in the energy range between $196 \mathrm{keV}$ and $2449 \mathrm{keV}$, that were located in a spectrum, acquired ten times longer that than the spectra the participants used for the analyses.

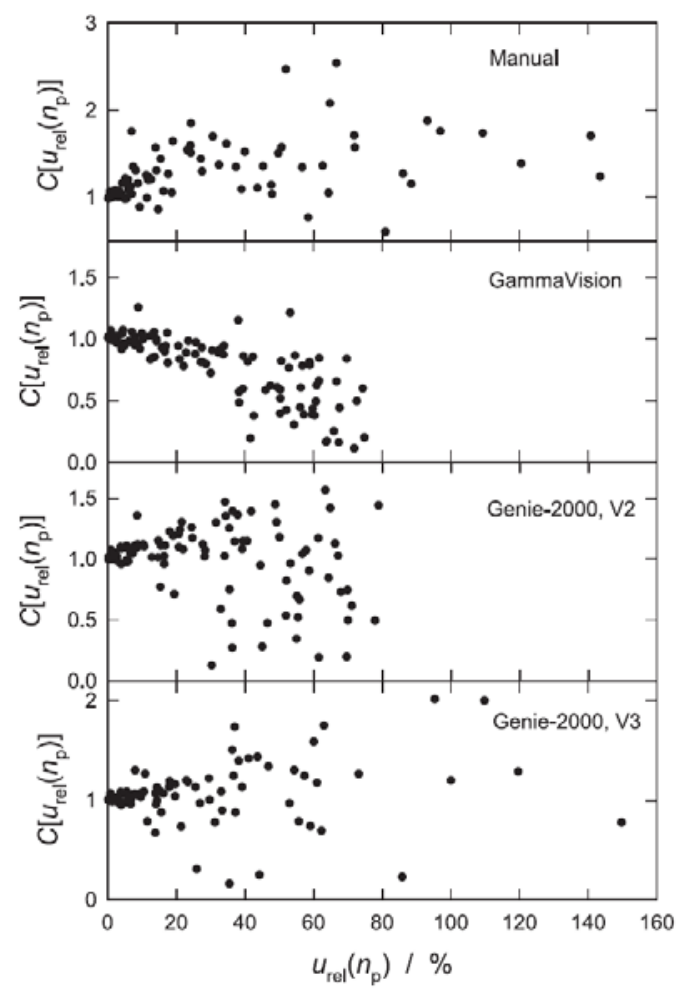

Fig. 1. Typical dependencies of the peak-specific correction factors on the relative uncertainty of the peak area.

\section{Analyses and methods}

The participating laboratories were advised to use a low value of the peak-significance parameter in order to locate and evaluate the peaks with a high relative peak-area uncertainty. Thirteen laboratories sent the results of fifteen peak analyses of the set of spectra they performed. Nine of them used Canberra's Genie-2000 Unidentified 2nd Differences program to locate the peaks and the Sum/Non-Linear LSQ Fit program (Canberra, 2001) to evaluate the peak areas. In these analyses the value of the peak-significance parameter was set between 2 and 3. One la- boratory sent three sets of analysis results obtained with the Genie- 2000 software, where the VMS Standard Peak Search Program (Canberra, 2006) was used for locating and evaluating the peaks, with the ANGES software (Mishev, 2016) and with the APTEC software (Aptec, 2000). One laboratory used the GammaVision software of Ortec (Ortec, 2016), one laboratory the software GAMMA-W of Dr. Westmeier GmbH (IAEA, 1998) and one laboratory reported results that were obtained manually, using the GammaVision software.

For each laboratory the mean correction factor and the mean re- lative uncertainty for each peak located in the spectra were calculated. The dependencies of the mean correction factor on the mean relative uncertainty for the data sets reported by the interested laboratories were compared and analysed. The spectra in six data formats and the excel 
file for calculating the means are available at https://www. dropbox.com/sh/f9ws2t6g5mnOnqd/AABiO5yq1ICf76EYdvMCZq6a? dl=0 for further use.

\section{Results}

It is a common feature of all datasets reported that the value of the correction factor $C(E)$ assumes a value of unity in the limit of small relative peak-area uncertainties. However, the range of relative un- certainties, where this factor remains near unity, i.e., where no cor- rection is necessary, differs greatly among datasets. In Fig. 1 the factor $\mathrm{C}[\mathrm{urel}(\mathrm{np})]$ is plotted as a function of the relative peak-area uncertainty for some examples of the results received, exhibiting different dependencies. The manual analysis shows a rapid increase in the correction factor for small relative uncertainties and then the correction factor remains constant; the analysis with Ortec's GammaVision software exhibits a steady decrease with the relative uncertainty; the analyses with Canberra's software exhibit a larger dispersion of correction factors, exhibiting a systematic decrease at high relative uncertainties (Genie- 2000 V2) and no systematic influence (Genie-2000 V3). Table 1 presents a short overview of the methods and the results reported.

4.

Discussion

It is obvious from Fig. 1 that the systematic influence at high relative uncertainties affects the correction factor in various ways. The datasets obtained by computer analysis show two regions of the relative uncertainties, where the dependence of the correction factor on the relative uncertainty differs: the region where the correction factor assumes values near unity and does not exhibit a dependence on the re- lative uncertainty, and a region where the correction factor is a decreasing function of the relative uncertainty. It can also be seen that in some datasets the region of the constant correction factor can extend up to the highest relative uncertainty reported. The dataset obtained manually exhibits a qualitatively different dependence.

\begin{tabular}{|c|c|c|c|c|c|}
\hline $\begin{array}{l}\text { No. of } \\
\text { data } \\
\text { set }\end{array}$ & $\begin{array}{l}\text { Method of } \\
\text { analysis }\end{array}$ & $\begin{array}{l}\text { No. of } \\
\text { spectra } \\
\text { analysed }\end{array}$ & $\begin{array}{l}\text { No. of } \\
\text { peak } \\
\text { energies }\end{array}$ & $u_{\text {rel,Max }} \%$ & $\begin{array}{l}\text { Maximum } \\
\text { relative } \\
\text { uncertainty } \\
\text { where no } \\
\text { correction is } \\
\text { needed } \%\end{array}$ \\
\hline 1 & Manual & 20 & 74 & 140 & 5 \\
\hline 2 & GAMMA-W & 35 & 97 & 50 & 30 \\
\hline 3 & GammaVision & 35 & 101 & 75 & 10 \\
\hline 4 & ANGES & 35 & 89 & 60 & 20 \\
\hline 5 & APTEC & 35 & 63 & 25 & 25 \\
\hline 6 & $\begin{array}{l}\text { Genie-2000, } \\
\text { VMS }\end{array}$ & 35 & 97 & 130 & 40 \\
\hline 7 & Genie-2000 & 35 & 78 & 130 & 130 \\
\hline 8 & Genie-2000 & 35 & 47 & 37 & 37 \\
\hline 9 & Genie-2000 & 35 & 96 & 80 & 30 \\
\hline 10 & Genie-2000 & 12 & 70 & 63 & 63 \\
\hline 11 & Genie-2000 & 35 & 81 & 42 & 20 \\
\hline 12 & Genie-2000 & 35 & 98 & 35 & 35 \\
\hline 13 & Genie-2000 & 35 & 79 & 145 & 145 \\
\hline 14 & Genie-2000 & 35 & 73 & 17 & - \\
\hline 15 & Genie-2000 & 35 & 99 & 600 & - \\
\hline
\end{tabular}

A large variability in the maximum relative uncertainties reported, urel,Max (Table 1), can be observed among the sets. Also, the maximum relative uncertainty where no correction of the peak area is needed differs among the sets of data reported. Fig. 2 presents the correlation between the maximum relative uncertainty reported and the relative uncertainty corresponding to the upper range of the interval where no influence of systematic effects on the correction factor can be observed. Three categories of datasets can be identified: the sets for which the upper limit of the range where no systematic influence occurs equals the maximum relative uncertainty, the sets where the upper limit is smaller than the maximum uncertainty reported, and the sets calculated with a sensitivity that does not allow us to make a conclusion about the systematic influence because in the peak analyses the peak-significance threshold was not set low enough to locate peaks having a relative uncertainty of the peak area that is high enough to show the systematic influence. 


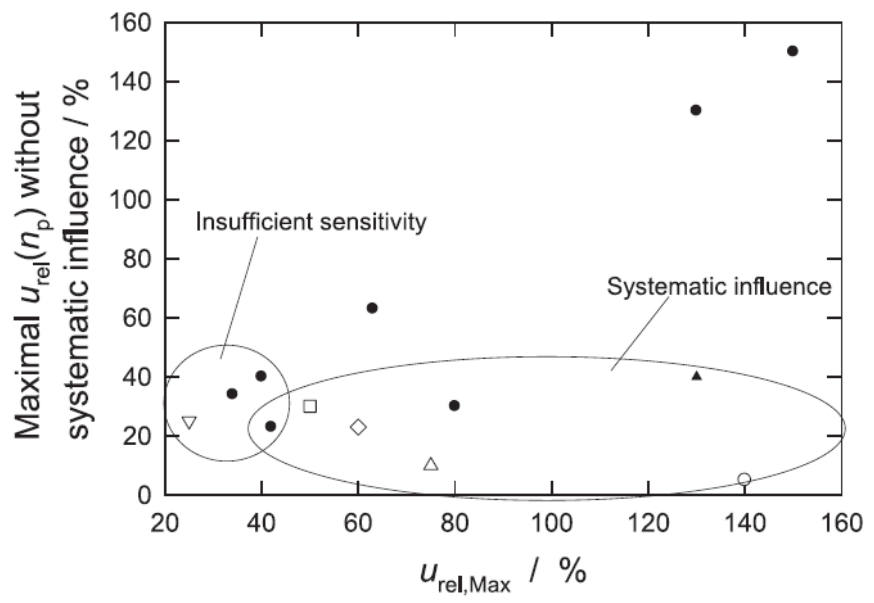

Fig. 2. The correlation between the maximum relative uncertainty reported and the upper limit of the range where no systematic influence on the correction factor can be observed. The symbols denote the method of analysis: • Genie-2000, $\boldsymbol{\Delta}$ Genie-2000 with VMS option, Omanual analysis, $\Delta$ GammaVision, $\square$ Gamma-W, $\triangle$ ANGES and APTEC.

The correlation between the number of peaks reported and the maximum relative uncertainty reported is presented in Fig. 3. It is expected that the datasets analysed with a lower peak significance threshold exhibit a larger number of reported peaks and a larger maximum reported relative uncertainty. However, the figure indicates that there is no simple correlation between the number of peaks and the maximum relative uncertainty. It is observed that three out of four datasets reporting maximum relative uncertainties in excess of $120 \%$ exhibit a relatively small number of reported peaks. This indicates that in these data sets a number of peaks are missing. To conclude about this, the number of peaks reported having a relative peak-area uncertainty in the upper $30 \%$ of the interval of relative uncertainties reported, i.e., an uncertainty larger than $70 \%$ of the maximum reported relative uncertainty, urel,Max, was counted. This number, expressed as a fraction of all the peaks detected, is presented in Table 2 and in Fig. 3 . It can be observed that the datasets with less than 80 peaks reported and a maximum relative uncertainty in excess of $120 \%$ exhibit the smallest fraction of peaks reported in the upper $30 \%$ of the interval of reported relative uncertainties. It follows that the peaks are missed predominantly from this interval. The three datasets with missing peaks were obtained with the manual analysis and the Genie-2000 V3 software.

Since in the two datasets obtained with the Genie-2000 V3 software no systematic influence on the correction factor was observed it seems that the version 3 of the Genie-2000 software tests peak-analysis results for reliability and rejects the peaks exhibiting the systematic influence. The dependence of the correction factor on the relative peak-area uncertainty was described with a linear function, assuming unity at the lower limit of the interval of relative uncertainties where the systematic influence occurs. This linear dependence represents the result of a smoothing of correction factors and is a property of the peak analysis. The slope of the linear function describes how strong the influence is and the uncertainty of the slope for the dispersion of the correction factors within the range where the correction occurs. Table 2 presents the data on the linear fits. The relative uncertainty of the slope describes the uncertainty of the linear dependence of the correction factor and affects the uncertainty of the peak areas, corrected for the systematic influence.

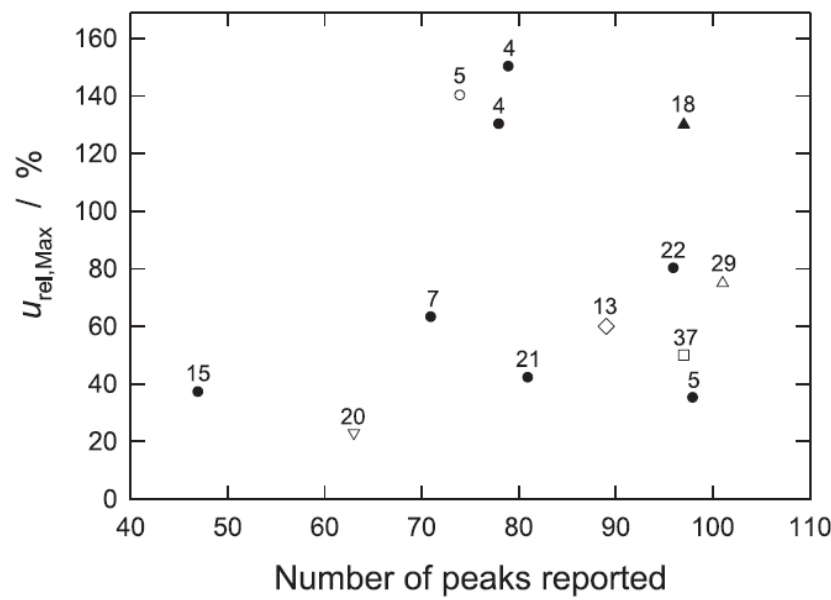

Fig. 3. The correlation between the number of peaks reported and the maximum relative uncertainty. The numbers by the symbols denote the fraction of peaks (in percent) with an uncertainty exceeding $0.7 u_{\text {rel,Max }}$. The methods of analysis are denoted with the same symbols as in Fig. 2. 
The effect of taking the systematic influence into account is presented in Fig. 4. Here the peak-specific correction factors are divided smooth linear dependence in the range of relative uncertainties, where the systematic influence occurs, are presented. It can be observed that the relative uncertainty of the slope for the dataset obtained with the Genie-2000 software (1.08) is reflected in the larger scattering of recalculated correction factors and a larger uncertainty of the correction as compared with the dataset obtained with the GammaVision software, where the relative uncertainty of the slope is 0.12 . The amount of information that is gained from the analysis of peaks having a large relative uncertainty can be assessed from the number of peaks having a relative peak-area uncertainty exceeding 0.7 urel,Max and the relative uncertainty of the slope, which determines the accuracy of the recalculation of the correction factors to a mean of unity in the region of the systematic influence. This correlation is presented in Fig. 5. It should be noted that datasets with a small fraction of peaks exceeding 0.7 urel,Max do not show the influence of the systematic effect so it is not possible to calculate the slope with a reasonable relative uncertainty and therefore they do not appear in Fig. 5. Since the information from the peak areas is larger when more peaks are reported with an uncertainty exceeding 0.7 urel,Max and when the relative un- certainty of the slope is smaller, it follows from the figure that at high relative uncertainties of the peak areas the most information can be obtained with the GammaVision and the Gamma-W software.

\begin{tabular}{|c|c|c|c|c|c|}
\hline $\begin{array}{l}\text { No. of } \\
\text { data set }\end{array}$ & $\begin{array}{l}\text { Maximum relative uncertainty } \\
\text { where no correction is needed / } \\
\%\end{array}$ & $\begin{array}{l}\text { Percent of peaks reported with a } \\
\text { relative uncertainty larger than } 70 \% \\
u_{\text {rel,Max }} \%\end{array}$ & Slope $/ \%^{-1}$ & $\begin{array}{l}\text { Relative uncertainty } \\
\text { of the slope }\end{array}$ & $\begin{array}{l}\text { Mean value and dispersion of correction } \\
\text { factors in the range where no correction is } \\
\text { needed }\end{array}$ \\
\hline 1 & 5 & 5 & 0 & - & - \\
\hline 2 & 30 & 37 & $-0.0362 \pm 0.0054$ & 0.15 & $1.01 \pm 0.08$ \\
\hline 3 & 10 & 29 & $-0.0091 \pm 0.0011$ & 0.12 & $1.01 \pm 0.07$ \\
\hline 4 & 20 & 13 & $-0.0160 \pm 0.0084$ & 0.52 & $1.03 \pm 0.06$ \\
\hline 5 & 25 & 22 & 0 & - & $1.01 \pm 0.21$ \\
\hline 6 & 40 & 18 & $-0.0027 \pm 0.0020$ & 0.74 & $0.99 \pm 0.17$ \\
\hline 7 & 130 & 4 & 0 & - & $1.07 \pm 0.35$ \\
\hline 8 & 37 & 15 & 0 & - & $1.07 \pm 0.19$ \\
\hline 9 & 30 & 22 & $-0.0040 \pm 0.0043$ & 1.08 & $1.07 \pm 0.11$ \\
\hline 10 & 63 & 9 & 0 & - & $1.00 \pm 0.20$ \\
\hline 11 & 20 & 21 & $-0.0250 \pm 0.0082$ & 0.32 & $1.02 \pm 0.11$ \\
\hline 12 & 35 & 5 & 0 & - & $1.04 \pm 0.23$ \\
\hline 13 & 145 & 4 & 0 & - & $1.05 \pm 0.34$ \\
\hline 14 & - & - & - & - & - \\
\hline 15 & - & - & - & - & - \\
\hline
\end{tabular}

The measure of the quality of the peak-analysis results where no systematic influence occurs, i.e., at relative peak-area uncertainties where the correction factors are near unity, is the mean of the correction factors in this range and the dispersion of the correction factors around the mean. These are presented in the last column of Table 2. Since in datasets where the peaks are missing a region where no correction is needed extends up to urel,Max, the dispersion of the correction factors increases. This occurs because the dispersion of correction fac- tors at large relative uncertainties escalates. The smallest dispersion of correction factors was obtained with the ANGES, GammaVision and Gamma-W software.

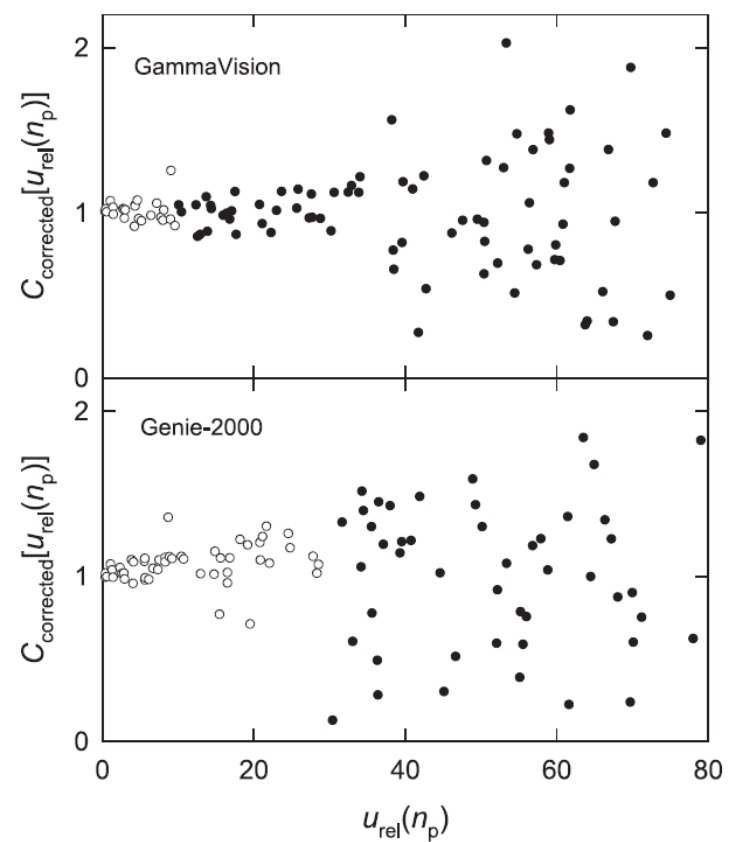

Fig. 4. The peak-specific correction factors divided by the linear dependence in the region of the systematic influence. Empty circles denote correction factors in the range of relative uncertainties where no systematic influences occur, full dots denote correction factors recalculated to the mean of unity in the range of the systematic influence. 
It should be mentioned that all the laboratories calculated a cor- rection factor that differs significantly from unity for the energy of $786 \mathrm{keV}$. The mean relative uncertainties reported for this peak are between $3.7 \%$ and $9.7 \%$, with a mean of $(7.4 \pm 0.5) \%$, and the re- ported correction factors lie in the interval from 1.22 to 1.35 with a mean of $1.28 \pm 0.01$. Since all the laboratories reported a correction factor that is too large, it can be concluded that the emission probability for gamma rays with energies near $786 \mathrm{keV}$ is too large.

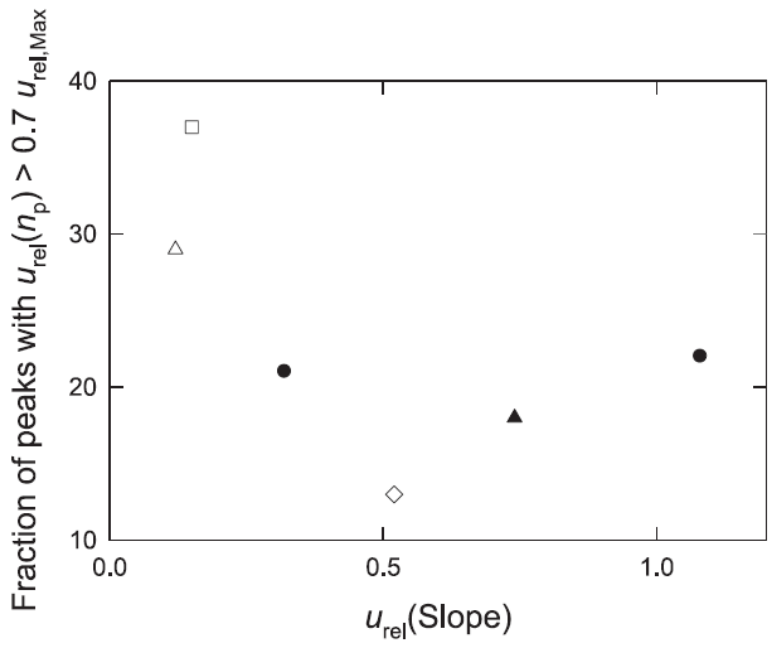

Fig. 5. The correlation between the relative uncertainty of the slope and the fraction of the peaks with $u_{\text {rel }}\left(n_{\mathrm{p}}\right)>0.7 u_{\text {rel,Max. }}$. The methods of analysis are denoted with the same symbols as in Fig. 2.

This emission probability originates in the intensities of gamma ray with energies of $785.96 \mathrm{keV}$ and $786.1 \mathrm{keV}$ with intensities of $(1.064 \pm 0.013) \%(\mathrm{k}=1)$ and $(0.31 \pm 0.05) \%(\mathrm{k}=1)$ from $214 \mathrm{~Pb}$ and $214 \mathrm{Bi}$, respectively (Nucleide-Lara on the web, 2016). The sum of the intensities, $(1.37 \pm 0.05) \%$, does not agree with the combined peak area in the spectrum. On the basis of the peak areas reported it is concluded that the sum of the intensities is too large for the factor of $1.28 \pm 0.01(k=1)$.

\section{Conclusions}

It was shown that sets of peak-analysis results that do not exhibit a systematic influence comprise very few peaks with a relative peak-area uncertainty in excess of 0.7 urel,Max. This prevents a reliable calculation of the influence of the systematic effect on the peak areas with a large uncertainty. Therefore, no conclusion about the systematic influence can be drawn here.

When the systematic influence is observed, it increases the areas of the peaks with a large relative uncertainty. The dispersion of the correction factors, corresponding to specific peaks, allows to calculating reliably only a linear dependence of the correction factors on the re- lative peak-area uncertainty.

Four parameters were extracted, which determine the quality of the peak-analysis results at high relative peak-area uncertainties: the range of the relative uncertainties where no correction is necessary, the scattering of correction factors around unity in the region where no correction is needed, the slope of the linear dependence, and its relative uncertainty. It was shown that the most reliable information about the peak areas with a large relative uncertainty was obtained with the GammaVision and the Gamma-W software. A similar conclusion was also drawn for the region of relative peak-area uncertainties where no correction is needed. Here, the smallest dispersion of correction factors was obtained by the ANGES, the GammaVision and the Gamma-W software. It can be concluded that the presented approach presents a new method for assessing the quality of the peak analysis software, which is designed for assessing systematic influences on the peak areas on a statistical basis.

\section{References}

Aptec, 2000. Aptec MCA Application Multichannel Analyzer version 7.00.01. Aptec Instruments Ltd. Canberra, 2001. Genie-2000 Customization Tools Manual. Canberra Industries Inc., Meriden. Canberra, 2006. Genie-2000 Spectroscopy Software Operations. Canberra Industries Inc., Meriden. Gilmore, R., 2008. Practical gamma-ray spectrometry, 2nd ed. John Wiley and Sons, Chichester, England. IAEA, 1998. IAEA-TECDOC-1011, Intercomparison of Gamma Ray Software Packages. IAEA, Vienna, Austria. 
Korun, M., Vidmar, T., Vodenik, B., 2008. Improving the reliability of peak evaluation results in gamma-ray spectrometry. Accredit. Qual. Assur. 13, 532-535.

Korun, M., Vodenik, B., Zorko, B., 2015. Reliability of the peak-analysis results in gamma- ray spectrometry for high relative peak-area uncertainties. Appl. Radiat. Isot. 105, 60-65.

Mishev, P., 2016. User Controlled Analysis of Gamma-ray Spectra, (http://www.iaea. org/inis/collection/NCLCollectionStore/ Public/33/017/33017172.pdf) (Accessed on 13 October 2016).

Nucleide-Lara on the web, 2016. 〈http://www.nucleide.org/Laraweb/), accessed on October 14, 2016.

ORTEC, 2016. GammaVision 8, 〈http://www.ortec-online.com/products/application-software/gammavision〉, accessed on January 23, 2017. 